BNL-114408-2017-JA

\title{
Probing Intermolecular Electron Delocalization in Dimer Radical Anions by Vibrational Spectroscopy
}

\author{
T. Mani, D. C. Grills \\ Submitted to Journal of Physical Chemistry B
}

August 2017

Chemistry Department

Brookhaven National Laboratory

\author{
U.S. Department of Energy \\ USDOE Office of Science (SC), \\ Basic Energy Sciences (BES) (SC-22)
}

\footnotetext{
Notice: This manuscript has been authored by employees of Brookhaven Science Associates, LLC under Contract No. DE- SC0012704 with the U.S. Department of Energy. The publisher by accepting the manuscript for publication acknowledges that the United States Government retains a non-exclusive, paidup, irrevocable, world-wide license to publish or reproduce the published form of this manuscript, or allow others to do so, for United States Government purposes.
} 


\section{DISCLAIMER}

This report was prepared as an account of work sponsored by an agency of the United States Government. Neither the United States Government nor any agency thereof, nor any of their employees, nor any of their contractors, subcontractors, or their employees, makes any warranty, express or implied, or assumes any legal liability or responsibility for the accuracy, completeness, or any third party's use or the results of such use of any information, apparatus, product, or process disclosed, or represents that its use would not infringe privately owned rights. Reference herein to any specific commercial product, process, or service by trade name, trademark, manufacturer, or otherwise, does not necessarily constitute or imply its endorsement, recommendation, or favoring by the United States Government or any agency thereof or its contractors or subcontractors. The views and opinions of authors expressed herein do not necessarily state or reflect those of the United States Government or any agency thereof. 


\title{
Probing Intermolecular Electron Delocalization in Dimer Radical Anions by Vibrational Spectroscopy
}

\author{
Tomoyasu Mani ${ }^{* \dagger}$ and David C. Grills
}

${ }^{\dagger}$ Department of Chemistry, University of Connecticut, Storrs, Connecticut 06269-3060, United States

* Chemistry Division, Brookhaven National Laboratory, Upton, New York 11973-5000, United States

\section{ABSTRACT}

Delocalization of charges is one of the factors controlling charge transport in conjugated molecules. It is considered to play an important role in the performance of a wide range of molecular technologies, including organic solar cells and organic electronics. Dimerization reactions are well-suited as a model to investigate intermolecular spatial delocalization of charges. While dimerization reactions of radical cations are well investigated, studies on radical anions are still scarce. Upon dimerization of radical anions with neutral counterparts, an electron is considered to delocalize over the two molecules. Here, by using time-resolved infrared (TRIR) detection coupled with pulse radiolysis, we show that radical anions of 4-n-hexyl-4'cyanobiphenyl (6CB) undergo dimerization reactions, with an electron equally delocalized over the two molecules. We have recently demonstrated that nitrile $v(\mathrm{C} \equiv \mathrm{N})$ vibrations respond to the 
degree of electron localization of nitrile-substituted anions: we can quantify the changes in the electronic charges from the neutral to the anion states in the nitriles by monitoring the $v(\mathrm{C} \equiv \mathrm{N}) \mathrm{IR}$ shifts. In the first part of this paper, we show that the sensitivity of the $v(\mathrm{C} \equiv \mathrm{N})$ IR shifts does not depend on solvent polarity. In the second part, we describe how probing the shifts of the nitrile IR vibrational band unambiguously confirms the formation of dimer radical anions, with $K_{\text {dim }}=3$ $\times 10^{4} \mathrm{M}^{-1}$. IR findings are corroborated by electronic absorption spectroscopy and electronic structure calculations. We find that the presence of a hexyl chain and the formation of $\pi-\pi$ interactions are both crucial for dimerization of radical anions of $6 \mathbf{C B}$. The present study provides clear evidence of spatial delocalization of electrons over two molecular fragments. 


\section{INTRODUCTION}

Electron delocalization is an important consideration in governing the energy and dynamics of charges in conjugated molecules and materials. ${ }^{1}$ Radical cations and anions can dimerize with neutral counterparts, resulting in a charge (either a hole or an electron) being delocalized over the two molecules. Such an intermolecular delocalization in dimers can serve as a suitable model to give insights into more complicated molecular systems such as those found in $\pi$-stacked organic materials, including semiconductors and solar cells. While dimerization reactions of radical cations are more common and have been well investigated for decades, ${ }^{2-8}$ studies on radical anions, including both intra- and intermolecular dimerizations, are still scarce at room temperature, ${ }^{9-11}$ and even at low temperature. ${ }^{12-19}$

Radiation-induced chemical reactions are widely used to study dimerization reactions of radicals as both radical cations and anions can be selectively generated and their behaviors observed spectroscopically. The appearance of charge resonance (CR) bands in UV-Vis-NIR spectra $^{11,14,18-19}$ is considered to be the main experimentally-observable characteristic property of dimer radical cations and anions. Such a CR absorption band in dimers is typically red-shifted from the monomer counterparts' absorption bands due to the delocalization of charges. While the observation of such a new band serves as a good diagnostic method, it often does not provide detailed information about the degree of charge delocalization in a dimerization process. Thus, due to the lack of a method to quantify delocalization of electrons, it has been difficult to study and clarify the exact nature of dimer radical anions.

Vibrational spectroscopy provides a superb sensitivity to the local molecular or surrounding environment. ${ }^{20}$ In recent years, it has become a powerful tool for studying electron dynamics of molecules in the condensed phase. ${ }^{21-23}$ Here, by using time-resolved infrared (TRIR) 
detection coupled with pulse radiolysis, ${ }^{24-25}$ we show that radical anions of 4-n-hexyl-4'cyanobiphenyl (6CB) undergo dimerization reactions with their neutral counterparts.

We recently demonstrated that nitrile $v(\mathrm{C} \equiv \mathrm{N})$ vibrations respond to the degree of electron localization of nitrile-substituted anions, and we constructed a calibration curve that connects the $v(\mathrm{C} \equiv \mathrm{N})$ IR shifts with the changes in the electronic charges from the neutral to the anion states in the nitriles. ${ }^{26}$ In the first part of this paper, we construct another calibration curve in a polar solvent, showing that the sensitivity of the $v(\mathrm{C} \equiv \mathrm{N})$ IR shift to the degree of electron delocalization is independent of solvent polarity. In the second part, we present a study of a dimerization reaction of radical anions of $6 \mathrm{CB}$, a class of liquid crystalline molecules, at room temperature. We observed this dimerization process by IR spectroscopy based on mid-infrared (mid-IR) spectral measurements in the region of the $v(\mathrm{C} \equiv \mathrm{N})$ band following pulse radiolysis. Using the constructed calibration curves, we provide unequivocal evidence for an intermolecular delocalization of the electron through the formation of dimers. Our observation was further supported by a measurement based on electronic absorption and electronic structure calculations. We discuss the effects of intermolecular geometry on the formation of dimer radical anions and intermolecular electron delocalization.

\section{EXPERIMENTAL METHODS}

2.1. General Information. All solvents and reagents used were obtained from standard commercial sources and used as received unless otherwise noted. FTIR spectra were recorded on a Thermo Nicolet Nexus 670 FTIR spectrometer using a cell equipped with $\mathrm{CaF}_{2}$ windows. Tetrahydrofuran (THF) was purified with a Vacuum Atmospheres Solvent Purifier System installed inside an inert atmosphere glovebox. 
2.2. Pulse Radiolysis. Pulse radiolysis experiments were performed at the Laser-Electron Accelerator Facility (LEAF) at Brookhaven National Laboratory. For UV-vis-NIR transient absorption detection, the experiments were performed as described before, ${ }^{27}$ using a quartz cell with a pathlength of $0.5 \mathrm{~cm}$. For TRIR detection, a detailed description of the experimental setup is given elsewhere. ${ }^{25}$ A home-built, airtight IR solution flow cell was used (1.10 mm pathlength), with $0.35 \mathrm{~mm}$ thick $\mathrm{CaF}_{2}$ windows. Continuous wave external-cavity quantum cascade lasers (Model 21047-MHF and Model 21043-MHF, Daylight Solutions, Inc.) were used as the IR probe source. The time resolution is limited to $\sim 40 \mathrm{~ns}$ in the current setup. Samples were dissolved in $N, N$-Dimethylformamide (DMF) or THF, and purged with argon. In some TRIR measurements, we observed baseline absorptions that amount to $\sim 10 \%$ of the peak absorptions. These baseline absorptions were subtracted from the reported spectra of the anions. The source of these absorptions may come from the solvents or minor products of solutes after radiolysis. Pulse radiolysis of DMF and THF has been used to generate radical anions of aromatic hydrocarbons. Equilibrium constants were determined by following an established method; ${ }^{28-29}$ an excess amount of biphenyl was added that rapidly captures the solvated electron, and then transfers an electron to $\mathbf{6 C B}$.

2.3. Computations. Computations were carried out with Gaussian09 D.01. ${ }^{30}$ The geometries were optimized with the B3LYP, ${ }^{31-32}$ LC- $\omega \mathrm{PBE}^{33-35}$ or $\omega \mathrm{B} 97 \mathrm{X}-\mathrm{D}^{36}$ functional in density functional theory (DFT) calculations. When noted, a dispersion correction $\left(\mathrm{GD} \mathrm{BJ}^{37}\right)$ is used with B3LYP and LC- $\omega$ PBE as implemented in Gaussian. The 6-31G(d) basis set was used unless otherwise noted. Polarizable continuum models $(\mathrm{PCM})^{38-40}$ for solvation were used. All calculations on anions were spin-unrestricted. All $n$-hexyl groups in oligofluorenes were replaced by ethyl groups. In some computations, $n$-hexyl groups in 6 CB were replaced by hydrogen. The 
geometry optimizations were performed without symmetry constraints. Frequency calculations were performed at the optimized geometries without anharmonicity corrections. Reported frequencies are not scaled. Enthalpies and Gibbs energies were obtained from single-point energies and thermal corrections from vibrational analyses; entropy corrections were scaled by a factor of 0.70 to account for decreased entropy in the condensed phase. ${ }^{41}$ Our scaling factor of 0.70 is based on the available experimental data for dimer cation formation of three aromatic hydrocarbons (benzene, naphthalene, and pyrene) in the condensed phase ${ }^{2}$ and the gas phase $\mathrm{e}^{42-43}$ (see Table S1, Supporting Information). Linear response time-dependent DFT (TD-DFT) calculations were performed for low-lying excited states to determine transition energies at the ground state geometries. The atomic charges were derived from a least-squares fit to the electrostatic potential calculated with the "Charges from electrostatic potentials using a Grid" (CHelpG) scheme ${ }^{44}$ as implemented in Gaussian09. The CHelpG scheme produces atomic charges that are fitted to reproduce the molecular electrostatic potential.

\section{RESULTS}

3.1. Calibration Curve. Radiolytic reduction of mononitrile-functionalized aryl compounds (PhCN, 1-NapCN, 9-AntCN, $\mathbf{F}_{\mathbf{n}} \mathbf{C N}$ where $\mathrm{n}=1-3$, structures are shown in Chart 1) in DMF $\left(D_{\mathrm{e}}=38.25\right)$ causes the $v(\mathrm{C} \equiv \mathrm{N})$ IR bands to shift to lower frequencies (Figure 1a and Figure S1, Supporting Information), in a similar manner to in $\operatorname{THF}\left(D_{\mathrm{e}}=7.52\right) .{ }^{45}$ Note that two peaks are observed for 1-NapCN-, likely due to Fermi resonance. ${ }^{46-47}$ The IR absorption spectral properties of the neutral and anionic species are summarized in Table 1. DFT calculations, using LC- $\omega$ PBE $\left(\omega=0.1\right.$ bohr $\left.^{-1}\right)$, support the trend in the IR frequencies, while the computed frequencies are systematically larger than the values experimentally observed. The LC- $\omega$ PBE $(\omega$ 
$=0.1 \mathrm{bohr}^{-1}$ ) functional was used as it can well reproduce the electronic properties of oligofluorenes. ${ }^{29,} 45,48 \mathrm{We}$ previously showed that anharmonicity corrections improved agreements with our experimental data, but $\Delta v$ did not improve. ${ }^{45}$

Chart 1. Molecular structures and abbreviations of mono-arylnitrile compounds and 4- $n$-hexyl4'-cyanobiphenyl $\left(\mathrm{R}=n-\mathrm{C}_{6} \mathrm{H}_{13}, \mathbf{6 C B}\right)$ and unsubstituted cyanobiphenyl $(\mathrm{R}=\mathrm{H}, \mathbf{B P C N})$.
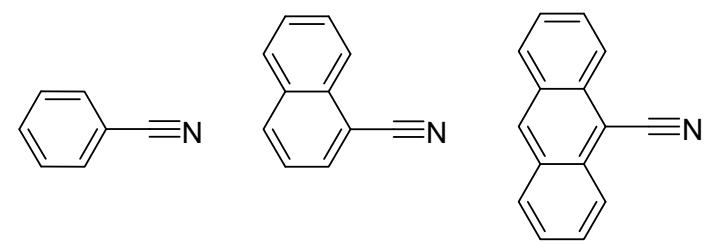

PhCN 1-NapCN 9-AntCN

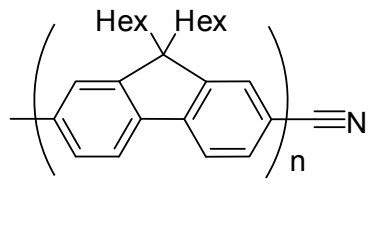

$\mathrm{F}_{\mathrm{n}} \mathrm{CN}$

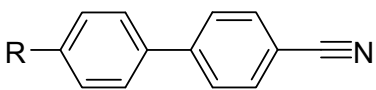

$6 \mathrm{CB}\left(\mathrm{R}=n-\mathrm{C}_{6} \mathrm{H}_{13}\right)$

Following the procedure described previously, ${ }^{45}$ a linear correlation was obtained between the observed changes in $v(\mathrm{C} \equiv \mathrm{N})$ IR absorbance $(\Delta v)$ and the changes in the computationally determined charges on the $\mathrm{CN}$ groups $(\Delta$ charge $)$ : $\Delta$ charge $=\left(q_{\mathrm{C}}+q_{\mathrm{N}}\right)_{\text {anion }}-$ $\left(q_{\mathrm{C}}+q_{\mathrm{N}}\right)_{\text {neutral }}$ except for the point 'Neutral' where $\Delta v$ and $\Delta$ charge are set to 0 . A calibration curve was constructed with the slope being $683 \pm 72 \mathrm{~cm}^{-1} / e$ in DMF (Figure 1b).
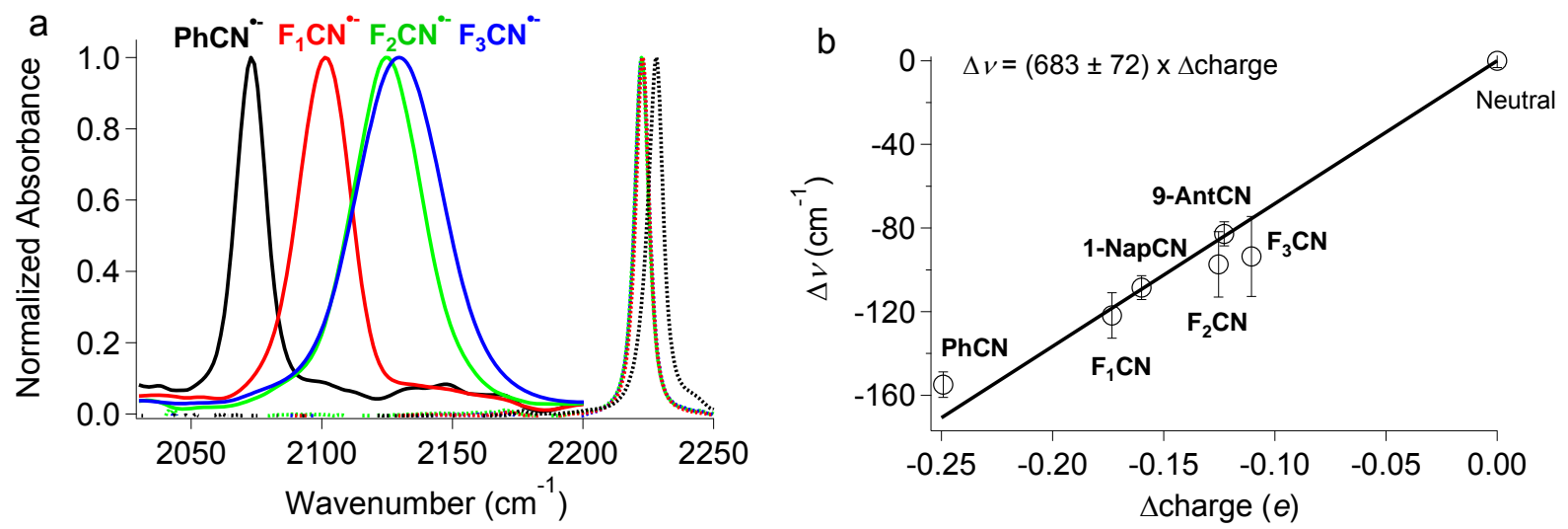
Figure 1. (a) FTIR spectra of neutral molecules $\mathbf{P h C N}, \mathbf{F}_{\mathbf{1}} \mathbf{C N}, \mathbf{F}_{2} \mathbf{C N}$, and $\mathbf{F}_{3} \mathbf{C N}$ in $\mathrm{DMF}$ solution (dotted lines) and TRIR spectra of their free anions obtained at a time delay of $\sim 100 \mathrm{~ns}$ after pulse radiolysis (solid lines). All spectra are baseline subtracted and normalized. (b) Calibration curve of the dependence of the experimentally-observed changes in $v(\mathrm{C} \equiv \mathrm{N})$ IR band position $(\Delta v)$ on the calculated changes in electrostatic potential CHelpG charges of the nitrile between the neutral and anion states ( $\Delta$ charge). The bars represent the full width half maximum (FWHM) of the anion peaks. A linear fit was obtained $\left(\mathrm{R}^{2}=0.95\right)$.

Table 1. Observed and Calculated $v(\mathrm{C} \equiv \mathrm{N})\left(\mathrm{cm}^{-1}\right)$ of Neutral and Anion Forms of Mononitrilefunctionalized Aryl Compounds in DMF.

\begin{tabular}{|c|c|c|c|c|c|c|c|c|}
\hline \multirow{3}{*}{ Name } & \multicolumn{3}{|c|}{ Neutral } & \multicolumn{3}{|c|}{ Anion } & \multicolumn{2}{|c|}{$\Delta v^{a}$} \\
\hline & \multicolumn{2}{|c|}{ Expt } & $\omega \mathrm{PBE}^{b}$ & \multicolumn{2}{|c|}{ Expt } & $\omega \mathrm{PBE}^{b}$ & \multirow[b]{2}{*}{ Expt } & \multirow[b]{2}{*}{$\omega \mathrm{PBE}^{b}$} \\
\hline & $v_{\max }$ & $\begin{array}{c}\mathrm{FWHM}^{c} \\
\left(\mathrm{~cm}^{-1}\right)\end{array}$ & $v_{\max }$ & $v_{\max }$ & $\begin{array}{c}\mathrm{FWHM}^{c} \\
\left(\mathrm{~cm}^{-1}\right)\end{array}$ & $v_{\max }$ & & \\
\hline PhCN & 2228 & 6.95 & 2307 & 2073 & 12.2 & 2096 & -155 & -210 \\
\hline $\mathrm{F}_{1} \mathrm{CN}$ & 2223 & 6.07 & 2299 & 2101 & 21.8 & 2124 & -122 & -175 \\
\hline $\mathrm{F}_{2} \mathrm{CN}$ & 2222 & 6.36 & 2299 & 2125 & 31.3 & 2145 & -97 & -154 \\
\hline $\mathrm{F}_{3} \mathrm{CN}$ & 2223 & 6.45 & 2299 & 2129 & 38.4 & 2149 & -94 & -150 \\
\hline 1-NapCN & 2222 & 6.98 & 2297 & $2114^{d}$ & $11.6^{d}$ & 2132 & -109 & -165 \\
\hline 9-AntCN & 2215 & 6.59 & 2282 & 2132 & 11.5 & 2166 & -83 & -116 \\
\hline
\end{tabular}

${ }^{a} \Delta v=v_{\text {anion }}-v_{\text {neutral. }}{ }^{b}$ The calculated frequencies are unscaled. All calculations used the LC$\omega \operatorname{PBE}\left(\omega=0.1 \mathrm{bohr}^{-1}\right)$ functional with the 6-31G(d) basis set and the PCM model for solvation in DMF. ${ }^{c}$ Full width half maximum (FWHM) was determined from the fitting of the spectra by a single Voigt function. The reported FWHM is of the Voigt profile. ${ }^{d}$ The peak corresponds to the higher energy and higher intensity band.

3.2. IR Band Shifts in Time. TRIR experiments producing the anions of 6CB $(\mathrm{c} \sim 20 \mathrm{mM}$, Chart 1) in DMF revealed a surprising spectroscopic change in the $v(\mathrm{C} \equiv \mathrm{N})$ region. 
A peak at $2106 \mathrm{~cm}^{-1}\left(\mathrm{FWHM}=18.8 \mathrm{~cm}^{-1}\right)$ was observed $\sim 40 \mathrm{~ns}$ after pulse radiolysis (Figure 2a). This peak (Peak 1) corresponds to $\mathbf{6 C B}^{\circ-}$ and the $v(\mathrm{C} \equiv \mathrm{N})$ IR shift from the neutral to the anion is explained based on the degree of electron delocalization ${ }^{45}$; as a comparison, $v(\mathrm{C} \equiv \mathrm{N})=$ $2110 \mathrm{~cm}^{-1}$ was observed for the anion of $\mathbf{F}_{\mathbf{1}} \mathbf{C N}$ (Table 1). ${ }^{26,49}$ The transient absorption of $\mathbf{6 C B} \mathbf{B}^{\bullet-}$ decayed on a time scale of less than a microsecond with the formation of a new, broader band at $v_{\max }=2164 \mathrm{~cm}^{-1}$ with FWHM $=27.8 \mathrm{~cm}^{-1}$ (Peak 2), as shown in Figure 2. A similar behavior was observed in the less polar solvent, THF (Table 2 and Figure S2, Supporting Information).
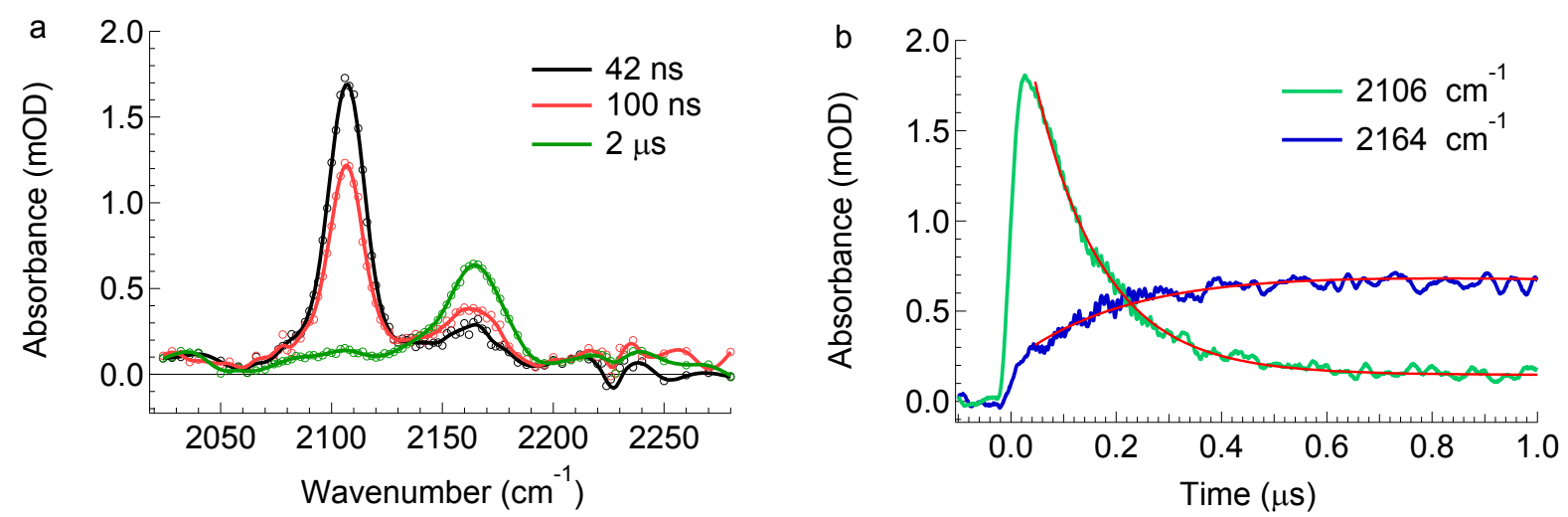

Figure 2. (a) TRIR spectra recorded at the specified time delays after pulse radiolysis of $\mathbf{6 C B}$ (c $\sim 20 \mathrm{mM}$ ) in DMF. The small negative peak around $2230 \mathrm{~cm}^{-1}$ is due to ground state bleaching of the neutral molecule. (b) Kinetic decay curves at 2106 and $2164 \mathrm{~cm}^{-1}$. The red lines are fitted curves.

Table 2. Observed $v(\mathrm{C} \equiv \mathrm{N})\left(\mathrm{cm}^{-1}\right)$ of Neutral and Anion Forms of 6CB in DMF and THF. 


\begin{tabular}{|c|c|c|c|c|c|c|c|c|c|}
\hline \multirow{2}{*}{ Solvent } & \multicolumn{2}{|c|}{ Neutral } & \multicolumn{3}{|c|}{$\begin{array}{l}\text { Anion Species } 1 \\
\text { Monomer }\end{array}$} & \multicolumn{3}{|c|}{$\begin{array}{c}\text { Anion Species } 2 \\
\text { Dimer }\end{array}$} & \multirow{2}{*}{$\begin{array}{c}\Delta v \\
\text { Ratio }^{\mathrm{c}}\end{array}$} \\
\hline & $v_{\max }$ & $\begin{array}{c}\mathrm{FWHM}^{a} \\
\left(\mathrm{~cm}^{-1}\right)\end{array}$ & $v_{\max }$ & $\begin{array}{c}\mathrm{FWHM}^{a} \\
\left(\mathrm{~cm}^{-1}\right)\end{array}$ & $\Delta v^{b}$ & $v_{\max }$ & $\begin{array}{l}\mathrm{FWHM}^{d} \\
\left(\mathrm{~cm}^{-1}\right)\end{array}$ & $\Delta v^{b}$ & \\
\hline DMF & 2226 & 6.72 & 2106 & 18.8 & -120 & 2164 & 27.8 & -62 & 1.94 \\
\hline THF & 2227 & 5.58 & 2114 & 11.7 & -113 & 2164 & 20.3 & -63 & 1.79 \\
\hline
\end{tabular}

${ }^{a}$ Full width half maximum (FWHM) was determined by the fitting of the spectra by a single Voigt function. The reported FWHM is of the Voigt profile. ${ }^{b} \Delta v=v_{\text {anion }}-v_{\text {neutral. }}{ }^{C}$ Ratio $=$ $\Delta v($ Species 1$) / \Delta v($ Species 2$)$.

The appearance of a second peak was not observed with non-alkylated cyanobiphenyl (BPCN), even at higher concentration (examined up to $\mathrm{c} \sim 100 \mathrm{mM}$, Figure 3). It was also not observed for $\mathrm{CBs}$ with shorter alkyl chains $\left(\mathrm{R}=\mathrm{CH}_{3}(\mathbf{1 C B})\right.$ and $n-\mathrm{C}_{3} \mathrm{H}_{9}(\mathbf{C C B})$ in Chart 1 , TRIR data not shown), nor with other previously studied aryl nitriles. ${ }^{45}$

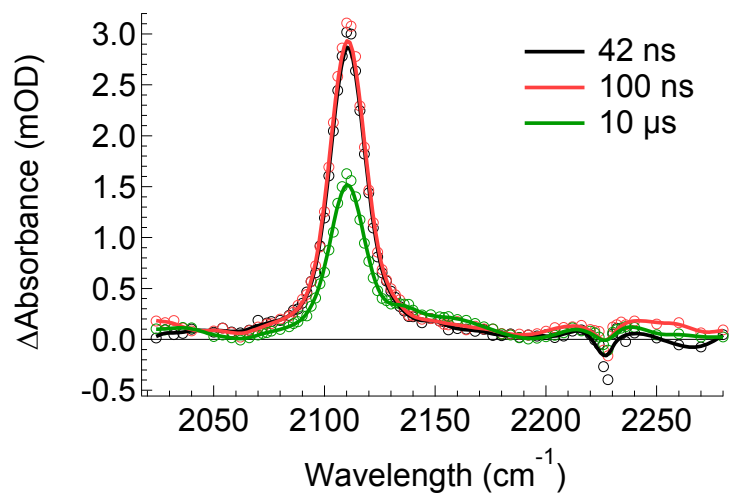

Figure 3. TRIR spectra recorded at the specified time delays after pulse radiolysis of BPCN (c $\sim 100 \mathrm{mM}$ ) in DMF. The small negative peak around $2230 \mathrm{~cm}^{-1}$ is due to ground state bleaching of the neutral molecule.

As described above and previously, ${ }^{45}$ the shift of $v(\mathrm{C} \equiv \mathrm{N})$ from neutral to anionic species reflects a change in the excess electron distribution in the nitrile group, or in other words the 
degree of electron localization. Therefore, we conclude that the new band corresponds to a dimer radical anion of $6 C B$ through

$$
6 \mathrm{CB}^{\circ-}+6 \mathrm{CB} \rightleftarrows(6 \mathrm{CB})_{2}{ }^{--}
$$

where the excess electron is shared by two $6 \mathbf{C B}$ molecules. We will further discuss the degree of charge delocalization in the dimer radical anion in Section 4.2.

\subsection{Charge Resonance Bands. A conventionally used criterion for dimer anion or} cation formation is the appearance of new bands in UV-Vis-NIR spectra, often as a charge resonance band, singly occupied molecular orbital $(\mathrm{SOMO}) \rightarrow \mathrm{SOMO}+1$ transition for anions and SOMO-1 $\rightarrow$ SOMO transition for cations. ${ }^{11,14,18-19}$ The time-resolved spectra in the vis/NIR region after pulse radiolysis of $\mathbf{6 C B}$ in DMF are shown in Figure 4a. Clearly, the initial absorption band of $6 \mathbf{C B}^{\circ}-\left(\lambda_{\max } \sim 660 \mathrm{~nm}\right)$ decays while a new band $\left(\lambda_{\max } \sim 920 \mathrm{~nm}\right)$ in the NIR range appears. The decay and formation rates observed in the vis/NIR range agree well with those observed in the mid-IR range (Figure 4b). A similar band formation was observed in THF (Figure S3, Supporting Information). DFT calculations support the assignment of the new absorption band at $920 \mathrm{~nm}$ to a dimer radical anion $(\mathbf{6 C B})_{2}{ }^{\circ}$, likely a charge resonance band (see below).
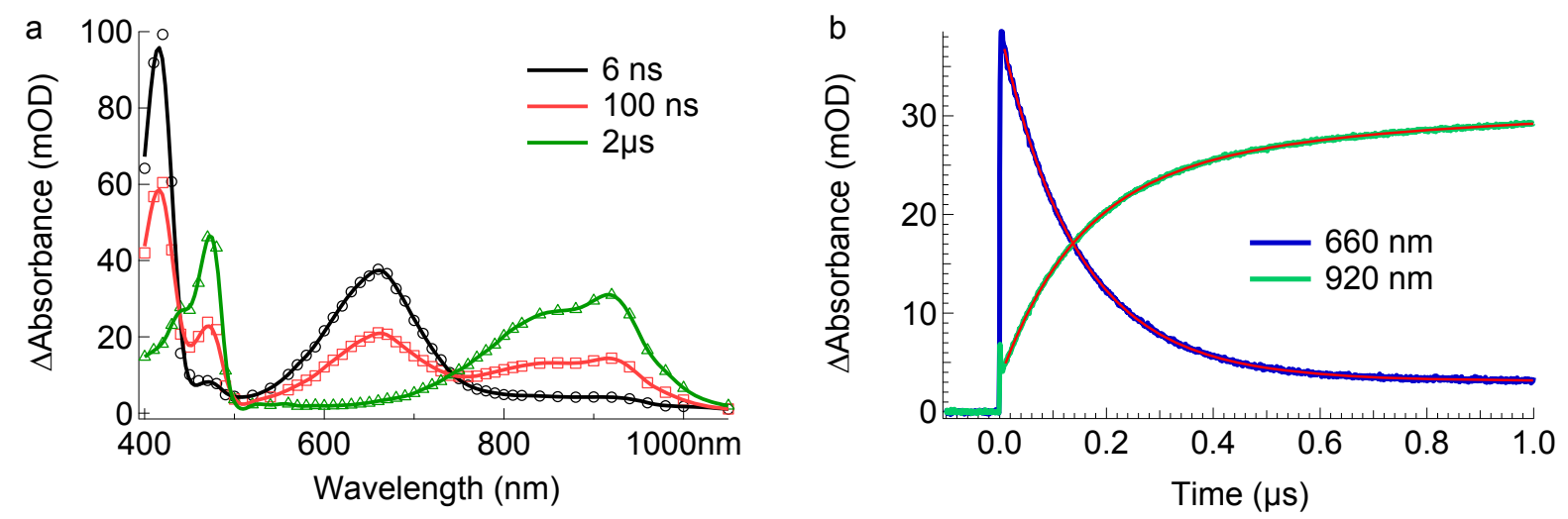
Figure 4. (a) Transient absorption spectra recorded at the specified time delays after pulse radiolysis of 6CB (c $\sim 20 \mathrm{mM}$ ) in DMF. (b) Kinetic decay curves of the transient absorptions at 660 and $920 \mathrm{~nm}$. The red lines are fitted curves.

\subsection{Rates and Association Constants of Dimerization}

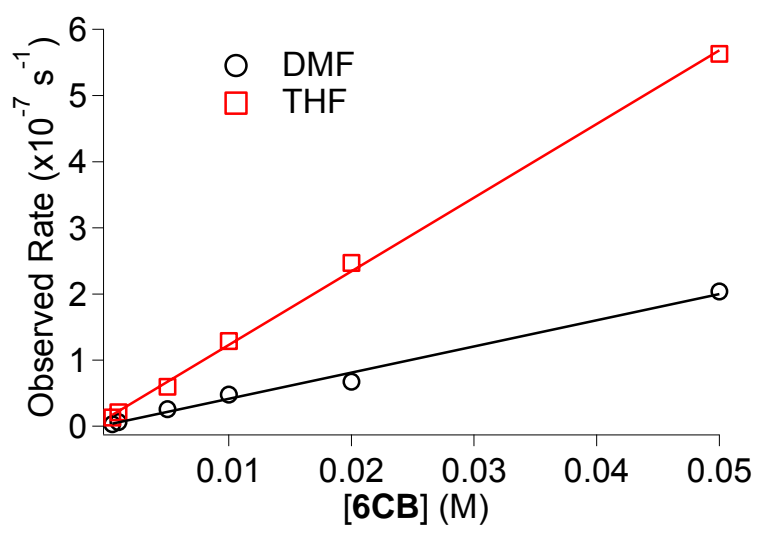

Figure 5. Plots of observed rate of dimerization of $6 \mathrm{CB}^{\circ-}$ with $6 \mathrm{CB}$ as a function of $[6 \mathrm{CB}]$ in DMF and THF, based on the growth of the NIR band of the dimer anion. The solid lines represent linear fits.

The bimolecular rate constants of dimerization $\left(k_{\mathrm{dim}}\right)$ of $\mathbf{6 C B}^{*-}$ with $6 \mathrm{CB}$ in DMF and THF were calculated to be $(4.0 \pm 0.2) \times 10^{8} \mathrm{M}^{-1} \mathrm{~s}^{-1}$ and $(1.1 \pm 0.1) \times 10^{9} \mathrm{M}^{-1} \mathrm{~s}^{-1}$, respectively, from kinetic measurements at six different concentrations of $6 \mathbf{C B}$, based on the observed rate constants (pseudo-first order) of the formation of dimer radical anions (Figure 5). The bimolecular rate constants were also determined based on the observed rate constants of the decay of monomer anions, giving comparable values (Table 3, Figure S4, Supporting Information). In THF, a solvent of low polarity, the dimerization reaction was slowed down by the presence of counter ions such as tetrabutylammonium ions that can form ion pairs with 
anions (Figure S5, Supporting Information). In the particular case of $0.1 \mathrm{M}$ tetrabutylammonium hexafluorophosphate $\left(\mathrm{TBA}^{+} \mathrm{PF}_{6}{ }^{-}\right)$, the dimerization becomes slower by a factor of about six.

Table 3. The bimolecular rate constants $\left(k_{\mathrm{dim}}\right)$ and Gibbs energy changes $\left(\Delta G_{\mathrm{dim}}\right)$ of dimerization of 6CB- $^{-*}$ with $6 \mathrm{CB}$ in DMF and THF.

\begin{tabular}{cccc}
\hline & \multicolumn{2}{c}{$k_{\operatorname{dim}}\left(\mathrm{M}^{-1} \mathrm{~s}^{-1}\right)$} & \\
& $\begin{array}{c}\text { From monitoring the } \\
\text { formation of (6CB) })_{2}^{--}\end{array}$ & $\begin{array}{c}\text { From monitoring } \\
\text { the decay of 6CB }\end{array}$ & $\Delta G_{\text {dim }}(\mathrm{eV})$ \\
\hline DMF & $(4.0 \pm 0.2) \times 10^{8}$ & $(3.7 \pm 0.2) \times 10^{8}$ & $-0.27 \pm 0.03$ \\
THF & $(1.1 \pm 0.1) \times 10^{9}$ & $(0.92 \pm 0.05) \times 10^{9}$ & $-0.27 \pm 0.02$ \\
\hline
\end{tabular}

At sufficiently low concentrations of $6 \mathbf{C B}$, equilibrium concentrations of $6 \mathbf{C B}^{\circ-}$ and $(6 \mathrm{CB})_{2}{ }^{-}$in reaction (1) can be spectroscopically observed. Thus, following an established method to measure the equilibrium constant by pulse radiolysis, ${ }^{28-29}$ we determined the equilibrium constant of reaction (1) ( $K_{\mathrm{dim}}$ ). The data at $\sim 650 \mathrm{~nm}$, corresponding to the monomer anion absorption, give $K_{\mathrm{dim}}=3.04 \times 10^{4} \mathrm{M}^{-1}(\mathrm{DMF})$ and $3.47 \times 10^{4} \mathrm{M}^{-1}$ (THF), from which the associated Gibbs energy changes $\left(\Delta G_{\mathrm{dim}}\right)$ were determined and tabulated in Table 3.

3.5. Computation of Dimer Radical Anions. Vibrational frequencies and thermochemical quantities were computed at selected levels of theory. LC- $\omega$ PBE $\left(\omega=0.1\right.$ bohr $\left.^{-1}\right)$ with GD3BJ dispersion correction yielded the results that were most consistent with the experimental values (Table S2, Supporting Information). The dispersion correction improves the agreement with the experimental data. Structurally, the dispersion correction makes the interplanar distance between the two 6CB molecules closer (Figure 6a and Figure S6, Supporting Information). 
Computed $v(\mathrm{C} \equiv \mathrm{N})$ bands of dimer radical anions are very sensitive to the conformation of the dimers. Among many conformations, two cases are considered; parallel and anti-parallel dimers whose optimized structures for $(\mathbf{6 C B})_{2}{ }^{-}$in DMF are shown in Figure $6 \mathrm{a}, \mathrm{b}$, and d, respectively. The two biphenyl rings are displaced from a complete face-to-face stacking in the optimized parallel $\left(\sim 20^{\circ}\right)$ and antiparallel $\left(\sim 11^{\circ}\right)$ conformations. Complete face-to-face stacked structures are not equilibrium structures. In the parallel conformation, the presence of $n$-hexyl chains displaced the two biphenyl rings in comparison with the absence of $n$-hexyl chains $\left(\sim 8^{\circ}\right.$ in $\left.(\mathbf{B P C N})_{2}{ }^{\circ}\right)$. The shortest interplanar distance between the two aryl rings is $\sim 3.4 \AA$ in both the parallel and antiparallel conformations, which is slightly shorter than the sum of the van der Waals radii for aromatic organic compounds $(3.45 \pm 0.05 \AA)^{50}$ and the interplanar distances observed in crystal structures of neutral 6CB $(\sim 3.56 \AA),{ }^{51}$ but longer than in the tetracyanoethylene dimer dianion, $(\text { TCNE })_{2}{ }^{2-}(2.89 \AA)$, and other computed structures of radical anions and cations such as the TCNE dimer radical anion, (TCNE) ${ }_{2}{ }^{-}(\sim 2.9 \AA),{ }^{52}$ and the benzene dimer radical cation $(\sim 3.2 \AA) .{ }^{53}$ It is also worthwhile to note that the dihedral angle in biphenyl decreases in the order of neutral $\left(\sim 34^{\circ}\right)>$ dimer radical anions $\left(\sim 21-23^{\circ}\right)>$ monomer radical anions $\left(\sim 13^{\circ}\right)$. This observation is consistent with, and further complements, the previous finding that the conjugated molecular plane becomes flatter to accommodate excess charges. ${ }^{54-55}$ 

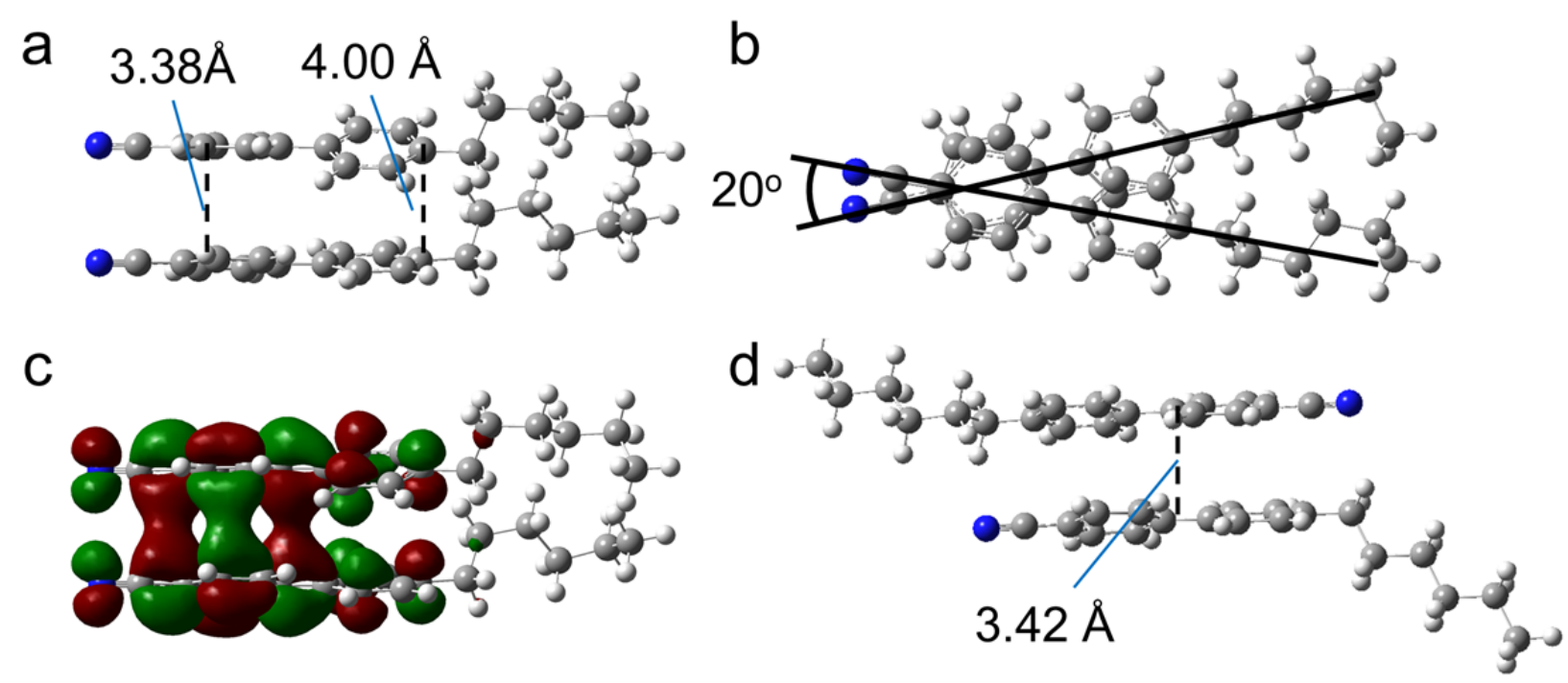

Figure 6. Optimized structures of $(\mathbf{6 C B})_{2}{ }^{--}$in DMF: (a) parallel conformation, (b) top view of the parallel conformation showing displacement of $20^{\circ}$ with respect to the two nitrile groups, (c) SOMO alpha orbital computed for the parallel conformation. (d) anti-parallel conformation.. All are computed with the LC- $\omega$ PBE $\left(\omega=0.1\right.$ bohr $\left.^{-1}\right)$ functional with the GD3BJ dispersion correction, using the 6-31G(d) basis set and the PCM model for solvation in DMF. All surfaces have an isovalue of 0.02 .

For both the parallel and anti-parallel dimer anions, there are two coupled $v(\mathrm{C} \equiv \mathrm{N})$ vibrational modes; one is symmetrical stretching and the other is asymmetrical stretching. The frequencies of the asymmetrical stretching mode are lower than those of the symmetrical stretching mode (Figure S7, Supporting Information). When the two molecules are not $\pi$-stacked, the excess electron is localized on one of the molecules, and thus a dimer radical anion is not formed (Figure S8, Supporting Information).

TD-DFT calculations showed that the dimer radical anion, $(\mathbf{6 C B})_{2}{ }^{\circ-}$, exhibits a red-shifted absorption band compared to the monomer anion, $\mathbf{6 C B}^{\circ}$, consistent with the experimental observation. The computed main lowest-energy transition $(\lambda \sim 1700 \mathrm{~nm}$ for parallel and $\sim 1400$ 
$\mathrm{nm}$ for antiparallel) in the dimer anions corresponds to the $\mathrm{SOMO} \rightarrow \mathrm{SOMO}+1$ transition with a low oscillator frequency. The next main transition band $(\lambda \sim 650 \mathrm{~nm})$ mainly corresponds to the $\mathrm{SOMO} \rightarrow \mathrm{SOMO}+6$ transition. This band is still red-shifted compared with the main transition of the anions (Figure S9, Supporting Information).

Gibbs energy changes $\left(\Delta G_{\mathrm{dim}}^{\mathrm{comp}}\right)$ associated with the formation of the dimer are computed based on the energies of the optimized structures of the neutral, monomer radical anion, and dimer radical anion, taking into account the thermal corrections. They are listed along with enthalpic changes $\Delta H_{\mathrm{dim}}^{\mathrm{comp}}$ and entropic changes $\Delta S_{\mathrm{dim}}^{\mathrm{comp}}$ in Table 4 . They are determined to be 0.14 and $-0.16 \mathrm{eV}$ for parallel and antiparallel conformations in DMF, and -0.19 and $-0.21 \mathrm{eV}$ for parallel and antiparallel conformations in THF at $298.15 \mathrm{~K}$, respectively. These values are more positive than the experimental values (Table 3). Computational results show that the dimer radical anion formation is thermodynamically favorable in both the non-alkylated BPCN and a shorter alkyl chained 1CB (Table S3, Supporting Information).

Table 4. Computed $\Delta H, \Delta S, \Delta G$, and $\Delta v(\mathrm{C} \equiv \mathrm{N})$ of the dimer radical anion formation of $\mathbf{6 C B}$ in parallel and anti-parallel configurations. ${ }^{a}$

\begin{tabular}{cccccc}
\hline \multirow{2}{*}{ Solvent } & Conformation & $\begin{array}{c}\Delta \boldsymbol{H}_{\mathbf{d i m}}^{\mathbf{c o m p}} \\
(\mathrm{eV})^{b}\end{array}$ & $\begin{array}{c}\Delta \boldsymbol{S}_{\mathbf{d i m}}^{\mathbf{c o m p}} \\
\left(\mathrm{cal} \mathrm{K}^{-1} \mathrm{~mol}^{-1}\right)^{c}\end{array}$ & $\begin{array}{c}\Delta \boldsymbol{G}_{\mathbf{d i m}}^{\text {Comp }} \\
(\mathrm{eV})^{b}\end{array}$ & $\begin{array}{c}\Delta v_{\text {comp }} \\
\left(\mathrm{cm}^{-1}\right)^{d}\end{array}$ \\
\hline \multirow{2}{*}{ DMF } & Parallel & -0.66 & -40.4 & -0.14 & -87 \\
\cline { 2 - 6 } & Antiparallel & -0.65 & -38.1 & -0.16 & -113 \\
\hline \multirow{2}{*}{ THF } & Parallel & -0.68 & -38.4 & -0.19 & -81 \\
\cline { 2 - 6 } & Antiparallel & -0.71 & -38.8 & -0.21 & -106
\end{tabular}

${ }^{a}$ All calculations used the LC- $\omega$ PBE $\left(\omega=0.1 \mathrm{bohr}^{-1}\right)$ functional with the GD3BJ dispersion correction, the 6-31G(d) basis set, and the PCM model for solvation in DMF and THF. ${ }^{b}$ At $298.15 \mathrm{~K} .{ }^{c}$ The reported values are scaled by $0.70 .{ }^{d}$ The calculated frequencies are unscaled. $\Delta v$ 
(monomer) $=-120 \mathrm{~cm}^{-1}$ and $-116 \mathrm{~cm}^{-1}$ for DMF and THF, respectively. $\Delta v$ is calculated from the weighted average of the two modes (symmetric and asymmetric stretching) in the dimers. Note that there is only one active mode (asymmetric stretching) in the anti-parallel configuration.

\section{DISCUSSION}

4.1. Nitrile Vibration as a Probe of Electron Delocalization. We constructed a calibration curve (Figure $1 \mathrm{~b}$ ) that connects the IR shift of $v(\mathrm{C} \equiv \mathrm{N})$ with the changes in electronic density distribution from the neutral to anionic forms of a series of $\mathrm{CN}$-functionalized aryl compounds in DMF, in order to complement a similar calibration curve we previously constructed in $\mathrm{THF}^{45}$ The slope of the calibration curve in DMF is similar to that in $\mathrm{THF}^{45}$ within the experimental error; $683 \pm 72 \mathrm{~cm}^{-1} / e(\mathrm{DMF})$ vs $668 \pm 18 \mathrm{~cm}^{-1} / e(\mathrm{THF})$. The larger uncertainty in DMF, compared to that in THF, likely comes from the broader line widths of the IR bands in DMF. The slope represents the sensitivity of $v(\mathrm{C} \equiv \mathrm{N})$ to the degree of electron delocalization. It also gives the expected shift for the hypothetical case when an electron is localized only on the nitrile, converting the bond order of $\mathrm{CN}$ from 3 to 2.5 . Our results show that the sensitivity, indicated by the slope, is independent of the solvent's polarity so that the calibration curves can be used in other solvents, signifying their usefulness in other relevant studies that use nitrile vibrations as a probe. It is worthwhile noting that the nitrile vibration of the anion is also sensitive to the presence of counter-ions and therefore the IR shift can be used to quantify ion pairing as well. ${ }^{26,}$ 49

The IR absorption linewidth of $v(\mathrm{C} \equiv \mathrm{N})$ is larger in DMF than we previously measured in THF. $^{45}$ The differences are small for the neutrals, but in some anionic cases, they are close to two times larger in DMF (e.g. for $\mathbf{F}_{\mathbf{1}} \mathbf{C N}, \mathrm{FWHM}=12.1 \mathrm{~cm}^{-1}$ in THF vs $21.8 \mathrm{~cm}^{-1}$ in DMF). A similar increase in linewidth in polar solvents was previously observed for $v(\mathrm{C} \equiv \mathrm{N})$ of neutral 
molecules. ${ }^{56-58}$ Such a broadening may stem from a faster vibrational relaxation, typically observed in polar solvents, ${ }^{59-61}$ and also suggests a more heterogeneous environment for $v(\mathrm{C} \equiv \mathrm{N})$ through various solute-solvent interactions in DMF.

\subsection{Dimerization}

4.2.1. IR Probe. Based on a linear relationship between the IR shift of $v(\mathrm{C} \equiv \mathrm{N})$ and $\Delta$ Charge, as evident from the calibration curves discussed above, the $\Delta v$ ratio of any two species directly gives the ratio of the excess electronic charge distributions on the nitriles of the respective molecular species. For the dimerization of $6 \mathbf{C B}^{-*}$ with $6 \mathrm{CB}$ (eq 1), the obtained ratios, $\frac{\Delta v(\text { Peak } 1)}{\Delta v(\text { Peak } 2)}=\frac{\Delta \text { Charge }(\text { Species } 1)}{\Delta \text { Charge }(\text { Species } 2)}$ are 1.94 (DMF) and 1.79 (THF) (Table 2), showing that the nitrile group in Species 1, corresponding to Peak 1, undergoes approximately twice as much change in the charge density on the $\mathrm{CN}$ group as that in Species 2, corresponding to Peak 2. This means that the excess electronic charge in Species 1 (monomer radical anion, $\mathbf{6 C B}^{\circ}$ ) is redistributed or delocalized equally to a neutral counterpart to become Species 2 (dimer radical anion, $(\mathbf{6 C B})_{2}{ }^{\circ}$ ), showing that it undergoes the dimerization reaction (Figure 7). The observation of the formation of a new red-shifted absorption band in the electronic absorption spectra, combined with TDDFT data, further supports the intermolecular delocalization of the electron.

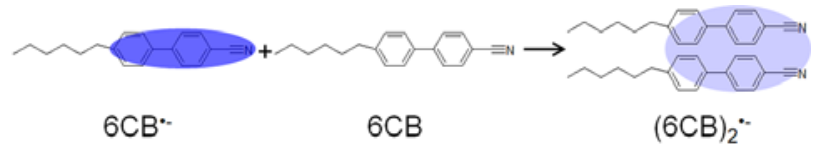

Figure 7. Intermolecular delocalization of an electron in a dimer radical anion of 6CB. The parallel conformation of the dimer is shown since it is the most likely structure in solution (see text).

4.2.2. Dimer Radical Anions.

4.2.1. Kinetics vs Thermodynamics. The bimolecular rate constant for dimer radical anion formation is three to four times higher in THF 
than in DMF (Table 3). The obtained value in DMF is similar to the values for the dimerization of bis(phenylethynyl)benzene radical anions observed by Majima and co-workers. ${ }^{19}$ The bimolecular rate constants (Table 3) are an order of magnitude smaller than the rate constant for a typical diffusion-controlled reaction $\left(\sim 10^{10} \mathrm{M}^{-1} \mathrm{~s}^{-1}\right)$. A dimer radical anion of $\mathbf{6 C B}$ is more thermodynamically stable than a monomer radical anion (Table 3), which is further supported by computations (see above). From a thermodynamic viewpoint, the dimerization process is equally favored in both solvents, suggesting that the dimerization reaction in these solvents is not thermodynamically controlled, but rather kinetically controlled. The striking difference in dimerization rate constants between a polar and nonpolar solvent can be explained by the reorganization of surrounding molecules that accompanies a dimerization process. The dimerization of radical anions results in the redistribution of an excess electron that requires a reorientation of the surrounding solvent molecules to accommodate the new charge distribution. Such a reorganization energy will be larger in polar DMF compared to the less polar THF, leading to a larger activation energy in polar DMF.

4.2.2.2. Success and Difficulties of Computations. The results of our computations qualitatively support our conclusion. Among the limited number of theory levels tested here (Table S2), we show that the inclusion of a GD3BJ dispersion correction makes the computed thermochemical quantities more reasonable, and that the combination of LC- $\omega$ PBE with GD3BJ yields the results that are most consistent with the experimental values. The importance of dispersion correction is well recognized for intermolecular interactions. ${ }^{62}$ Our computational results show that the formation of dimer radical anions is enthalpically favorable to a large degree $\left(\Delta H_{\mathrm{dim}}^{\mathrm{comp}} \sim-0.6-0.7 \mathrm{eV}\right)$. This large enthalpic gain is offset by the entropic contributions, giving the overall $\Delta G_{\mathrm{dim}}^{\mathrm{comp}} \sim-0.15-0.20 \mathrm{eV}$. We believe that the computed entropy term, even 
after the solvation correction, is still large; about two times larger than the reported experimental values for cation radical dimers (Table S1, Supporting Information). ${ }^{2}$ An antiparallel conformation is slightly more thermodynamically stable than a parallel conformation within thermal energy at room temperature $\left(k_{\mathrm{B}} \mathrm{T} \sim 25 \mathrm{meV}\right)$. TD-DFT calculations qualitatively predict the red-shifted absorption observed in dimer radical anions compared to that in the monomer. Poor quantitative agreements in the electronic transition energies between the computational (both parallel and antiparallel) and experimental values may indicate that the LC- $\omega$ PBE functional is not optimal for this system, or that other conformations should be considered. However, our current computational thermochemical and TD-DFT data generally support the formation of dimer radical anions.

Vibrational frequency is another parameter that we have experimental data to compare with. In the (6CB) ${ }_{2}{ }^{-}$dimer radical anion, two distinct bands corresponding to two different $v(\mathrm{C} \equiv \mathrm{N})$ vibrational modes (coupled symmetrical and asymmetrical stretching) are expected. For example, in a $\pi$-stacked parallel conformation, the computation predicted a $2158 \mathrm{~cm}^{-1}$ band for asymmetrical stretching and a $2196 \mathrm{~cm}^{-1}$ band for symmetrical stretching, while in a $\pi$-stacked antiparallel conformation, the computation predicted a $2151 \mathrm{~cm}^{-1}$ band for asymmetrical stretching and a $2194 \mathrm{~cm}^{-1}$ band for symmetrical stretching. The symmetrical stretching mode in the antiparallel conformation is not IR active; therefore, the predicted intensity is very low (Figure S7, Supporting Information). Based on a linear IR calibration curve, and as we observed experimentally, we would expect the computed IR shift, $\Delta v_{\text {comp }}$, for the dimer radical anion to be half of $\Delta v_{\text {comp }}$ for the monomer radical anion; in DMF $\Delta v_{\text {comp }}$ (monomer) $=-120 \mathrm{~cm}^{-1}$, therefore the expected $\Delta v_{\text {comp }}($ dimer $)=-60 \mathrm{~cm}^{-1}$. Given the large linewidth of the $v(\mathrm{C} \equiv \mathrm{N})$ band in solution, the two calculated bands in the parallel conformation could merge into one broad band ( $\Delta v_{\text {comp }}=$ 
$\left.-87 \mathrm{~cm}^{-1}\right)$ to align with the experimentally observed single, broad $v(\mathrm{C} \equiv \mathrm{N})$ band. On the other hand, in the antiparallel conformation, the IR active asymmetric stretching mode gives $\Delta v_{\text {comp }}=$ $113 \mathrm{~cm}^{-1}$, which is a much larger discrepancy relative to the expected $\Delta v_{\text {comp }}$ (dimer) of $-60 \mathrm{~cm}^{-1}$. Therefore, we can conclude that the frequency calculation result for the parallel conformation is qualitatively closer to this simplistic picture.

Our computational study, though currently limited in scope, illustrates the difficulty in describing radical anion dimers using DFT. With the limited amount of experimental data available, little efforts were so far made to describe the electronic structures of such systems while radical cation dimers and charge-transfer complexes have been the topics of extensive studies. ${ }^{63-65} \mathrm{We}$ anticipate that more experimental studies, including the current one, will allow chemists to further investigate this interesting class of species computationally.

\subsubsection{Importance of Alkyl Chains.}

Given that both the parallel and antiparallel conformations are more or less equally stable in terms of computational thermodynamics, based on our experimental data and frequency calculations, we expect that the dimer anions likely adopt parallel conformations with displaced aromatic planes. However, at this stage, it is not possible to rule out the possibility of antiparallel and other conformations, and further studies are needed to clarify this point. In a parallel conformation, the electron density is more localized on the aromatic ring on the side of the nitriles with some amount between the two molecules $(\alpha$ orbital of SOMO, Figure 6c). This also manifests itself in a closer interplanar distance between the two aromatic rings with the nitrile, compared to that between the rings with the alkyl chain (Figure 6a). Inspection of low frequency vibrational modes reveals some modes with substantial intermolecular character. Many modes appear to be mixed with other "single-molecule" vibrational modes, such as those in the $n$-hexyl chains. Some representative modes are shown in 
Figure S10, Supporting Information. The existence of such vibrational modes indicates that there might exist a bond formation between the two 6 CBs as shown in the case of (TCNE) ${ }_{2}{ }^{2-}{ }^{26}$

While we do not have a crystal structure of the dimer radical anions, the case of neutral 6CB can provide some insights. The crystal structure of $6 C B$ shows that the $6 C B$ molecules are packed in such a way that the $\mathrm{CN}$ groups are antiparallel between neighboring molecules, ${ }^{51}$ due

to electrostatic interactions. ${ }^{67-68}$ In the current case of dimer radical anions, the dimerization reactions take place in a diluted solution where the 6CB molecules are not likely to form neutral dimers prior to pulse radiolysis, which is further supported by the absence of the $(\mathbf{6 C B})_{2}{ }^{\circ-}$ peak at early time. In addition, non-alkylated cyanobiphenyl or shorter alkyl-chains do not undergo dimerization reactions at room temperature. This indicates that the dimerization reaction of $\mathbf{6 C B}^{--}$is not purely governed by the stabilization resulting from increasing the spatial distribution of an excess charge, but that it is also assisted in part by hydrophobic interactions of the $n$-hexyl chains. Such interactions may decrease the entropic cost of dimer radical anion formation. Thus, $\pi-\pi$ stacking alone will not drive intermolecular electron delocalization, signifying the importance of molecular geometry on intermolecular electron delocalization.

\section{CONCLUSIONS}

In conclusion, we have presented another demonstration of the superb sensitivity of the nitrile vibration to the degree of electron delocalization. In the first part, we showed that the sensitivity of the nitrile vibration is mostly an intrinsic property of the nitrile group, independent of solvent polarity. Thus, calibration curves that connect the IR shift of $v(C \equiv N)$ with the changes in electronic density distribution from the neutral to anionic forms can be applied to various molecular systems to study the degree of electron delocalization. In the second part, we used this 
sensitivity of the nitrile to study a new and unique case of dimer radical anion formation of 4- $n$ hexyl-4'-cyanobiphenyl that occurs at room temperature. IR shifts of the nitrile vibration unambiguously show that an excess electron charge becomes equally distributed over the two molecules. Our IR study, combined with conventional electronic absorption spectroscopy and electronic structure calculations, could elucidate some details of the electronic structure of the dimer radical anions. We conclude that the presence of a long alkyl chain and the formation of $\pi$ $\pi$ interactions both play crucial roles in the dimerization of the radical anions of $6 \mathbf{C B}$. 


\section{ASSOCIATED CONTENT}

Supporting Information. The Supporting Information is available free of charge on the ACS Publications website at DOI: 10.1021/acs.jpcb.XXXX. Figures S1-10 and Tables S1-3 (PDF) and coordinates for aromatic nitriles (PhCN, 1-NapCN, 9-AntCN, $\mathbf{F}_{\mathbf{n}} \mathbf{C N}(\mathrm{n}=1-3)$, and $\left.\mathbf{6 C B}\right)$ as xyz files (ZIP).

\section{AUTHOR INFORMATION}

\section{Corresponding Author}

* tomoyasu.mani@uconn.edu

\section{ACKNOWLEDGMENT}

Part of this work, and use of the LEAF facility of the BNL Accelerator Center for Energy Research and the computer Cluster at the Center for Functional Nanomaterials, was supported by the U.S. Department of Energy, Office of Science, Office of Basic Energy Sciences, Division of Chemical Sciences, Geosciences \& Biosciences under contract no. DE-SC0012704. T.M. is grateful for the startup funds at the University of Connecticut. We thank Dr. Mehmed Z. Ertem at BNL for helpful discussions about the DFT calculations. 
TOC Graphic

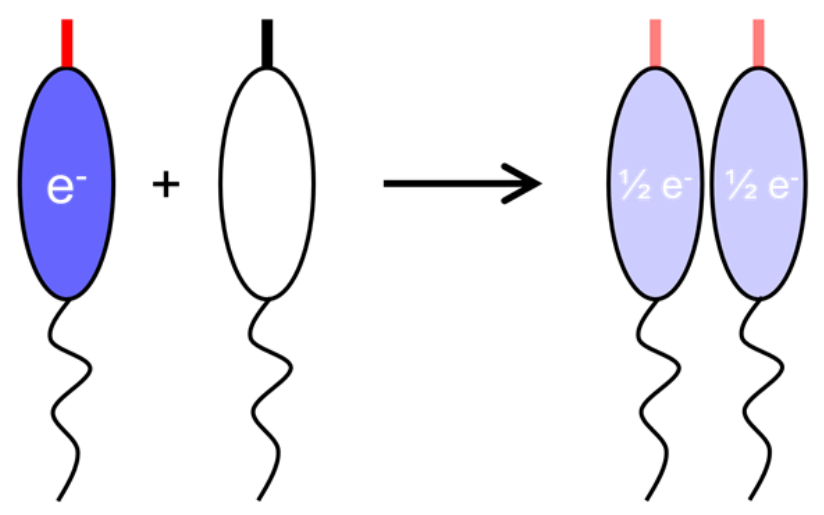




\section{REFERENCES}

1. Clarke, T. M.; Durrant, J. R., Charge Photogeneration in Organic Solar Cells. Chem. Rev. 2010, 110 (11), 6736-6767.

2. Badger, B.; Brocklehurst, B., Formation of Dimer Cations of Aromatic Hydrocarbons. Nature 1968, 219 (5151), 263-263.

3. Symons, M. C. R., Radical cations in condensed phases. Chem. Soc. Rev. 1984, 13 (4), 393-439.

4. Tsuchida, A.; Tsujii, Y.; Ito, S.; Yamamoto, M.; Wada, Y., Conformations of naphthalene dimer cation radicals studies by laser photolysis. J. Phys. Chem. 1989, 93 (4), 12441248.

5. Inokuchi, Y.; Ohashi, K.; Matsumoto, M.; Nishi, N., Photodissociation Spectrum of Naphthalene Dimer Cation. J. Phys. Chem. 1995, 99 (11), 3416-3418.

6. Small, D.; Zaitsev, V.; Jung, Y.; Rosokha, S. V.; Head-Gordon, M.; Kochi, J. K., Intermolecular $\pi$-to- $\pi$ Bonding between Stacked Aromatic Dyads. Experimental and Theoretical Binding Energies and Near-IR Optical Transitions for Phenalenyl Radical/Radical versus Radical/Cation Dimerizations. J. Am. Chem. Soc. 2004, 126 (42), 13850-13858.

7. Golder, M. R.; Wong, B. M.; Jasti, R., Photophysical and theoretical investigations of the [8]cycloparaphenylene radical cation and its charge-resonance dimer. Chemical Science 2013, 4 (11), 4285-4291.

8. Attah, I. K.; Platt, S. P.; Meot-Ner, M.; El-Shall, M. S.; Peverati, R.; Head-Gordon, M., What Is the Structure of the Naphthalene-Benzene Heterodimer Radical Cation? Binding Energy, Charge Delocalization, and Unexpected Charge-Transfer Interaction in Stacked Dimer and Trimer Radical Cations. J. Phys. Chem. Lett. 2015, 6 (7), 1111-1118. 
9. Majima, T.; Tojo, S.; Takamuku, S., Formation of Dimer Radical Anions of Aromatic Acetylenes during Pulse Radiolysis and $\gamma$-Radiolysis. J. Phys. Chem. A 1997, 101 (6), 10481055.

10. Ichinose, N.; Hobo, J.; Tojo, S.; Majima, T., Observation of intramolecular dimer radical anion of 1,1-diarylmethanols bearing electron withdrawing groups at room temperature. Chem. Phys. Lett. 2000, 330 (1-2), 97-102.

11. Fujitsuka, M.; Samori, S.; Tojo, S.; Haley, M. M.; Majima, T., Dimerization Reaction of Regioisomeric Bis(phenylethynyl)benzene Radical Anions during Pulse Radiolysis. ChemPlusChem 2012, 77 (8), 682-687.

12. Dadley, D.; Evans, A. G., Reactions of radical anions. Part IV. The dimerisation of the tolan radical anion. J. Chem. Soc. B 1967, 418-422.

13. Ishitani, A.; Nagakura, S., Electronic absorption and E.S.R. spectra of the benzene and paracyclophane anions. Mol. Phys. 1967, 12 (1), 1-12.

14. Shida, T.; Iwata, S., Absorption Spectra of Dianthracene Anion Radical and Anthracene Dimer Anion. J. Chem. Phys. 1972, 56 (6), 2858-2864.

15. Arai, S.; Kira, A.; Imamura, M., Low-temperature pulse radiolysis and .gamma.irradiated matrix studies of dimer anions of olefin derivatives. J. Phys. Chem. 1977, 81 (2), 110113.

16. Kira, A.; Ishiwata, M.; Imamura, M.; Tabata, Y., Absorption spectra of acrylonitrile monomer and dimer anions as studied by a rigid-matrix method. Radiat Phys Chem 1980, 15 (6), $663-665$. 
17. Ganesan, V.; Rosokha, S. V.; Kochi, J. K., Isolation of the Latent Precursor Complex in Electron-Transfer Dynamics. Intermolecular Association and Self-Exchange with Acceptor Anion Radicals. J. Am. Chem. Soc. 2003, 125 (9), 2559-2571.

18. Fujitsuka, M.; Tojo, S.; Shinmyozu, T.; Majima, T., Intramolecular dimer radical anions of [3n] cyclophanes: transannular distance dependent stabilization energy. Chemical Communications 2009, (12), 1553-1555.

19. Tojo, S.; Fujitsuka, M.; Majima, T., Intramolecular Charge Resonance in Dimer Radical Anions of Di-, Tri-, Tetra-, and Pentaphenylalkanes. J. Org. Chem. 2012, 77 (11), 4932-4938.

20. Fried, S. D.; Boxer, S. G., Measuring Electric Fields and Noncovalent Interactions Using the Vibrational Stark Effect. Accounts Chem. Res. 2015, 48 (4), 998-1006.

21. Asbury, J., Ultrafast Infrared Probes of Electronic Processes in Materials. In Ultrafast Infrared Vibrational Spectroscopy, CRC Press: 2013; pp 239-268.

22. Vlček, A.; Kvapilová, H.; Towrie, M.; Záliš, S., Electron-Transfer Acceleration Investigated by Time Resolved Infrared Spectroscopy. Accounts Chem. Res. 2015, 48 (3), 868876.

23. Dereka, B.; Koch, M.; Vauthey, E., Looking at Photoinduced Charge Transfer Processes in the IR: Answers to Several Long-Standing Questions. Accounts Chem. Res. 2017, 50 (2), 426434.

24. Grills, D. C.; Cook, A. R.; Fujita, E.; George, M. W.; Preses, J. M.; Wishart, J. F., Application of External-Cavity Quantum Cascade Infrared Lasers to Nanosecond Time-Resolved Infrared Spectroscopy of Condensed-Phase Samples Following Pulse Radiolysis. Appl. Spectrosc. 2010, 64 (6), 563-570. 
25. Grills, D. C.; Farrington, J. A.; Layne, B. H.; Preses, J. M.; Bernstein, H. J.; Wishart, J. F., Development of nanosecond time-resolved infrared detection at the LEAF pulse radiolysis facility. Rev. Sci. Instrum. 2015, 86 (4), 044102.

26. Hack, J.; Grills, D. C.; Miller, J. R.; Mani, T., Identification of Ion-Pair Structures in Solution by Vibrational Stark Effects. J. Phys. Chem. B 2016, 120 (6), 1149-1157.

27. Wishart, J. F.; Cook, A. R.; Miller, J. R., The LEAF picosecond pulse radiolysis facility at Brookhaven National Laboratory. Rev. Sci. Instrum. 2004, 75 (11), 4359-4366.

28. Takeda, N.; Asaoka, S.; Miller, J. R., Nature and energies of electrons and holes in a conjugated polymer, polyfluorene. J. Am. Chem. Soc. 2006, 128 (50), 16073-16082.

29. Zaikowski, L.; Kaur, P.; Gelfond, C.; Selvaggio, E.; Asaoka, S.; Wu, Q.; Chen, H. C.; Takeda, N.; Cook, A. R.; Yang, A., et al., Polarons, Bipolarons, and Side-By-Side Polarons in Reduction of Oligofluorenes. J. Am. Chem. Soc. 2012, 134 (26), 10852-10863.

30. Frisch, M. J.; Trucks, G. W.; Schlegel, H. B.; Scuseria, G. E.; Robb, M. A.; Cheeseman, J. R.; Scalmani, G.; Barone, V.; Mennucci, B.; Petersson, G. A., et al. Gaussian 09, Gaussian, Inc.: Wallingford, CT, USA, 2009.

31. Becke, A. D., Density-Functional Thermochemistry. III. The Role of Exact Exchange. $J$. Chem. Phys. 1993, 98 (7), 5648-5652.

32. Stephens, P. J.; Devlin, F. J.; Chabalowski, C. F.; Frisch, M. J., Ab-Initio Calculation of Vibrational Absorption and Circular-Dichroism Spectra Using Density-Functional Force-Fields. J. Phys. Chem. 1994, 98 (45), 11623-11627.

33. Vydrov, O. A.; Heyd, J.; Krukau, A. V.; Scuseria, G. E., Importance of short-range versus long-range Hartree-Fock exchange for the performance of hybrid density functionals. $J$. Chem. Phys. 2006, 125 (7), 074106. 
34. Vydrov, O. A.; Scuseria, G. E., Assessment of a long-range corrected hybrid functional. J. Chem. Phys. 2006, 125 (23), 234109.

35. Vydrov, O. A.; Scuseria, G. E.; Perdew, J. P., Tests of functionals for systems with fractional electron number. J. Chem. Phys. 2007, 126 (15), 154109 1-9.

36. Chai, J.-D.; Head-Gordon, M., Long-range corrected hybrid density functionals with damped atom-atom dispersion corrections. Phys. Chem. Chem. Phys. 2008, 10 (44), 6615-6620.

37. Grimme, S.; Ehrlich, S.; Goerigk, L., Effect of the damping function in dispersion corrected density functional theory. J. Comput. Chem. 2011, 32 (7), 1456-1465.

38. Tomasi, J.; Mennucci, B.; Cammi, R., Quantum mechanical continuum solvation models. Chem. Rev. 2005, 105 (8), 2999-3093.

39. Improta, R.; Barone, V.; Scalmani, G.; Frisch, M. J., A state-specific polarizable continuum model time dependent density functional theory method for excited state calculations in solution. J. Chem. Phys. 2006, 125 (5), 054103 1-9.

40. Improta, R.; Scalmani, G.; Frisch, M. J.; Barone, V., Toward effective and reliable fluorescence energies in solution by a new state specific polarizable continuum model time dependent density functional theory approach. J. Chem. Phys. 2007, 127 (7), 074504 1-9.

41. Tobisch, S.; Ziegler, T., Catalytic Oligomerization of Ethylene to Higher Linear $\alpha-$ Olefins Promoted by the Cationic Group 4 [(†5-Cp-(CMe2-bridge)-Ph)MII(ethylene)2]+ $(\mathrm{M}=$ Ti, Zr, Hf) Active Catalysts: A Density Functional Investigation of the Influence of the Metal on the Catalytic Activity and Selectivity. J. Am. Chem. Soc. 2004, 126 (29), 9059-9071.

42. Meot-Ner, M., Dimer cations of polycyclic aromatics. Experimental bonding energies and resonance stabilization. J. Phys. Chem. 1980, 84 (21), 2724-2728. 
43. Rusyniak, M.; Ibrahim, Y.; Alsharaeh, E.; Meot, N.; El-Shall, M. S., Mass-Selected Ion Mobility Studies of the Isomerization of the Benzene Radical Cation and Binding Energy of the Benzene Dimer Cation. Separation of Isomeric Ions by Dimer Formation. The Journal of Physical Chemistry A 2003, 107 (38), 7656-7666.

44. Breneman, C. M.; Wiberg, K. B., Determining Atom-Centered Monopoles from Molecular Electrostatic Potentials - the Need for High Sampling Density in Formamide Conformational-Analysis. J. Comput. Chem. 1990, 11 (3), 361-373.

45. Mani, T.; Grills, D. C.; Newton, M. D.; Miller, J. R., Electron Localization of Anions Probed by Nitrile Vibrations. J. Am. Chem. Soc. 2015, 137 (34), 10979-10991.

46. Nyquist, R. A., Solvent-Induced Nitrile Frequency Shifts: Acetonitrile and Benzonitrile. Appl. Spectrosc. 1990, 44 (8), 1405-1407.

47. Andrews, S. S.; Boxer, S. G., Vibrational stark effects of nitriles I. Methods and experimental results. J. Phys. Chem. A 2000, 104 (51), 11853-11863.

48. Zaikowski, L.; Mauro, G.; Bird, M.; Karten, B.; Asaoka, S.; Wu, Q.; Cook, A. R.; Miller, J. R., Charge Transfer Fluorescence and $34 \mathrm{~nm}$ Exciton Diffusion Length in Polymers with Electron Acceptor End Traps. J. Phys. Chem. B 2015, 119 (24), 7231-7241.

49. Mani, T.; Grills, D. C.; Miller, J. R., Vibrational Stark Effects To Identify Ion Pairing and Determine Reduction Potentials in Electrolyte-Free Environments. J. Am. Chem. Soc. 2015, 137 (3), 1136-1140.

50. Miller, J. S., Four-Center Carbon-Carbon Bonding. Accounts Chem. Res. 2007, 40 (3), 189-196.

51. Kuribayashi, M.; Hori, K., Crystal structures of 4-cyano-4-hexylbiphenyl (6CB) and 4cyano-4-heptylbiphenyl (7CB) in relation to odd-even effects. Liq. Cryst. 1999, 26 (6), 809-815. 
52. Wang, Q.; Newton, M. D., Structure, Energetics, and Electronic Coupling in the (TCNE2)-• Encounter Complex in Solution: A Polarizable Continuum Study. J. Phys. Chem. B 2008, $112(2), 568-576$.

53. Pieniazek, P. A.; Krylov, A. I.; Bradforth, S. E., Electronic structure of the benzene dimer cation. J. Chem. Phys. 2007, 127 (4), 044317.

54. Fratiloiu, S.; Grozema, F. C.; Koizumi, Y.; Seki, S.; Saeki, A.; Tagawa, S.; Dudek, S. P.; Siebbeles, L. D. A., Electronic Structure and Optical Properties of Charged Oligofluorenes Studied by VIS/NIR Spectroscopy and Time-Dependent Density Functional Theory. J. Phys. Chem. B 2006, 110 (12), 5984-5993.

55. Mani, T.; Miller, J. R., Role of Bad Dihedral Angles: Methylfluorenes Act as Energy Barriers for Excitons and Polarons of Oligofluorenes. J. Phys. Chem. A 2014, 118 (40), 94519459.

56. Getahun, Z.; Huang, C.-Y.; Wang, T.; De León, B.; DeGrado, W. F.; Gai, F., Using Nitrile-Derivatized Amino Acids as Infrared Probes of Local Environment. J. Am. Chem. Soc. 2003, 125 (2), 405-411.

57. Levinson, N. M.; Fried, S. D.; Boxer, S. G., Solvent-Induced Infrared Frequency Shifts in Aromatic Nitriles Are Quantitatively Described by the Vibrational Stark Effect. J. Phys. Chem. B 2012, 116 (35), 10470-10476.

58. Maienschein-Cline, M. G.; Londergan, C. H., The CN Stretching Band of Aliphatic Thiocyanate is Sensitive to Solvent Dynamics and Specific Solvation. J. Phys. Chem. A 2007, $111(40), 10020-10025$.

59. Bakker, H. J.; Planken, P. C. M.; Lagendijk, A., Role of solvent on vibrational energy transfer in solution. Nature 1990, 347 (6295), 745-747. 
60. Park, K.-H.; Jeon, J.; Park, Y.; Lee, S.; Kwon, H.-J.; Joo, C.; Park, S.; Han, H.; Cho, M., Infrared Probes Based on Nitrile-Derivatized Prolines: Thermal Insulation Effect and Enhanced Dynamic Range. J. Phys. Chem. Lett. 2013, 4 (13), 2105-2110.

61. van Wilderen, L. J. G. W.; Kern-Michler, D.; Muller-Werkmeister, H. M.; Bredenbeck, J., Vibrational dynamics and solvatochromism of the label SCN in various solvents and hemoglobin by time dependent IR and 2D-IR spectroscopy. Phys. Chem. Chem. Phys. 2014, 16 (36), 19643-19653.

62. Grimme, S., Density functional theory with London dispersion corrections. WIREs Comput. Mol. Sci. 2011, 1 (2), 211-228.

63. Steinmann, S. N.; Corminboeuf, C., Exploring the Limits of Density Functional Approximations for Interaction Energies of Molecular Precursors to Organic Electronics. $J$. Chem. Theory Comput. 2012, 8 (11), 4305-4316.

64. Steinmann, S. N.; Piemontesi, C.; Delachat, A.; Corminboeuf, C., Why are the Interaction Energies of Charge-Transfer Complexes Challenging for DFT? J. Chem. Theory Comput. 2012, $8(5), 1629-1640$.

65. Corminboeuf, C., Minimizing Density Functional Failures for Non-Covalent Interactions Beyond van der Waals Complexes. Accounts Chem. Res. 2014, 47 (11), 3217-3224.

66. Casado, J.; Burrezo, P. M.; Ramírez, F. J.; Navarrete, J. T. L.; Lapidus, S. H.; Stephens, P. W.; Vo, H.-L.; Miller, J. S.; Mota, F.; Novoa, J. J., Evidence for Multicenter Bonding in Dianionic Tetracyanoethylene Dimers by Raman Spectroscopy. Angew. Chem. Int. Edit. 2013, $52(25), 6421-6425$. 
67. Amovilli, C.; Cacelli, I.; Campanile, S.; Prampolini, G., Calculation of the intermolecular energy of large molecules by a fragmentation scheme: Application to the 4-n-pentyl-4'cyanobiphenyl (5CB) dimer. J. Chem. Phys. 2002, 117 (7), 3003-3012.

68. Zhang, J.; Guo, H., Transferability of Coarse-Grained Force Field for nCB Liquid Crystal Systems. J. Phys. Chem. B 2014, 118 (17), 4647-4660. 